\title{
Autophagy inhibition enhances therapy-induced apoptosis in a Myc-induced model of lymphoma
}

\author{
Ravi K. Amaravadi, ${ }^{1,2}$ Duonan Yu, ${ }^{3}$ Julian J. Lum, ${ }^{2}$ Thi Bui, ${ }^{2}$ Maria A. Christophorou, ${ }^{4}$ \\ Gerard I. Evan, ${ }^{4}$ Andrei Thomas-Tikhonenko, ${ }^{3}$ and Craig B. Thompson ${ }^{1,2}$ \\ 1Division of Hematology/Oncology, Department of Medicine, ${ }^{2}$ Department of Cancer Biology, Abramson Family Cancer Research Institute, and \\ ${ }^{3}$ Department of Pathobiology, University of Pennsylvania, Philadelphia, Pennsylvania, USA. ${ }^{4}$ Cancer Research Institute, \\ University of California San Francisco Comprehensive Cancer Center, San Francisco, California, USA.
}

\begin{abstract}
Autophagy is a lysosome-dependent degradative pathway frequently activated in tumor cells treated with chemotherapy or radiation. Whether autophagy observed in treated cancer cells represents a mechanism that allows tumor cells to survive therapy or a mechanism for initiating a nonapoptotic form of programmed cell death remains controversial. To address this issue, the role of autophagy in a $M y c$-induced model of $1 \mathrm{ym}$ phoma generated from cells derived from $p 53 E R^{T A M} / p 53 E R^{T A M}$ mice (with $E R$ denoting estrogen receptor) was examined. Such tumors are resistant to apoptosis due to a lack of nuclear p53. Systemic administration of tamoxifen led to $\mathrm{p} 53$ activation and tumor regression followed by tumor recurrence. Activation of p53 was associated with the rapid appearance of apoptotic cells and the induction of autophagy in surviving cells. Inhibition of autophagy with either chloroquine or ATG5 short hairpin RNA (shRNA) enhanced the ability of either $\mathrm{p} 53$ activation or alkylating drug therapy to induce tumor cell death. These studies provide evidence that autophagy serves as a survival pathway in tumor cells treated with apoptosis activators and a rationale for the use of autophagy inhibitors such as chloroquine in combination with therapies designed to induce apoptosis in human cancers.
\end{abstract}

\section{Introduction}

Macroautophagy (referred to hereafter as autophagy) is a process conserved by evolution that allows cells to sequester cytoplasmic contents through the formation of double-membrane vesicles (autophagosomes) and target them for degradation through the fusion of autophagosomes with lysosomes, creating single-membrane autolysosomes. A number of antineoplastic therapies have been observed to induce autophagy in human cancer cell lines (1-4). Whether autophagy induced by therapy contributes to tumor cell death or represents a mechanism of resistance to therapy-mediated cell death remains uncertain. Two observations that argue in favor of autophagy as a reflection of the therapeutic efficacy of antineoplastic agents are (a) that persistent activation of autophagy can lead to programmed cell death (5) and (b) that the autophagy regulator beclin 1 (BECN1) is a haploinsufficient tumorsuppressor gene that induces autophagy when overexpressed (6). These findings suggest that stimulation of autophagy may be detrimental to cancer cells and that therapies that inhibit autophagy may lead to enhanced tumor growth.

Accumulating evidence suggests that autophagy can also represent an adaptive strategy by which cells clear damaged organelles and survive bioenergetic stress. Autophagy, by targeting cytoplasmic proteins and organelles for lysosomal degradation, plays a role in recycling organelles and proteins that may be damaged by

Nonstandard abbreviations used: $B E C N 1$, beclin 1; CQ, chloroquine; ER, estrogen receptor; h2, hairpin 2; h7, hairpin 7; HC, hairpin control; HCQ, hydroxychloroquine; hTAM, 4-hydroxytamoxifen; LC3, mammalian homolog of yeast Atg8; MEF, mouse embryonic fibroblast; MNNG, $\mathrm{N}$-methyl-N'-nitro-N-nitrosoguanidine; shRNA, short hairpin RNA; TAM, tamoxifen.

Conflict of interest: The authors have declared that no conflict of interest exists. Citation for this article: J. Clin. Invest. 117:326-336 (2007). doi:10.1172/JCI28833. increased reactive oxygen species generated by the cellular stress associated with activated oncogenes and cancer therapies $(7,8)$. Autophagy also promotes the survival of cells resistant to apoptosis when they are deprived of extracellular nutrients or growth factors. Treatment of such cells dependent on autophagy for survival with the drug chloroquine (CQ) results in brisk cell death (9).

CQ is a lysosomotropic drug that raises intralysosomal $\mathrm{pH}(10)$ and impairs autophagic protein degradation (11). By blocking the last step of the autophagy pathway, either CQ treatment (9) or knockdown of the lysosomal protein LAMP-2 (12) leads to the accumulation of ineffective autophagosomes and cell death in cells reliant on autophagy for survival. Due to its favorable therapeutic index in animals, CQ could be used as a tool to investigate the role of autophagy as a response to therapeutic stress in tumors grown in mice.

To test the effect of CQ and its modulation of tumor cell autophagy in vivo, a mouse model of B cell lymphoma was generated. This model utilizes a p53-estrogen receptor knockin mouse $\left(p 53 E R^{T A M} / p 53 E R^{T A M}\right)$ that allows for the in vivo temporal dissection of the effects of p53 activation (13). Bone marrow cells from these mice were infected in vivo with a Myc-expressing retrovirus at high multiplicity in the flanks of recipient mice. This reproducibly gave rise to polyclonal $M y c / p 53 E R^{T A M}$ lymphomas with a B cell phenotype. These tumors can be utilized in therapeutic studies because of their ability to be adoptively transferred to the flanks of syngeneic mice. In the absence of therapy, the resulting tumors grow rapidly, as they are effectively Myc positive, $p 53$ null. Upon systemic administration of tamoxifen (TAM), the p53ER fusion protein translocates to the nucleus, restoring p53 function and initiating apoptosis and tumor regression. After a period of tumor latency, $100 \%$ of animals experience tumor recurrence despite continuous TAM treatment. 


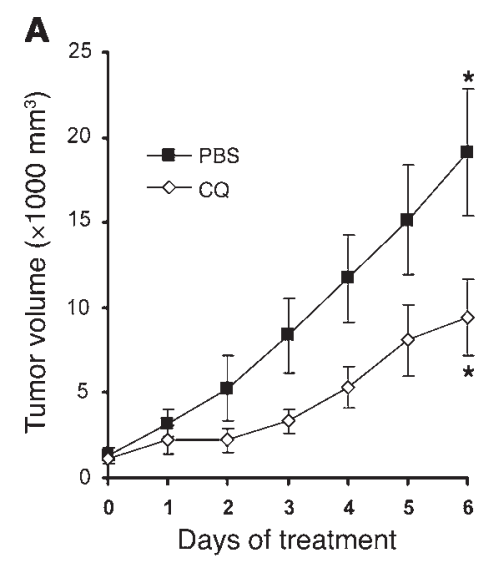

B

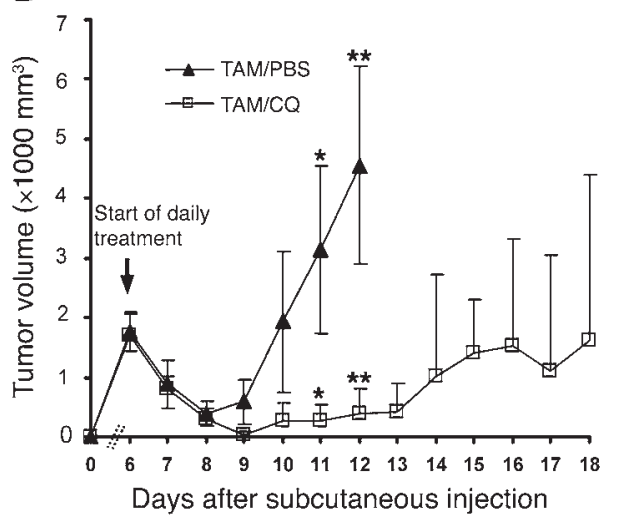

\section{Figure 1}

Effects of $C Q$ with and without p53 activation on the regression of Myc/p53ERTAM lymphomas. (A) CQ impairs tumor growth. Cells from a primary Myc/p53ER TAM tumor were harvested and passaged in vivo in 6 syngeneic C57BL/6×129F1 mice. Cells were injected subcutaneously into the flanks of mice. When tumors reached a volume of more than $1,000 \mathrm{~mm}^{3}$, mice were assigned to daily PBS i.p. or $60 \mathrm{mg} / \mathrm{kg} / \mathrm{d} C Q$ i.p. Results shown are mean \pm SD daily tumor volumes and are representative of multiple experiments. ${ }^{*} P<0.05$. (B) CQ delays tumor recurrence after $\mathrm{p53}$-induced tumor regression. Myc/p53ER ${ }^{\text {TAM }}$ cells were injected subcutaneously into the flanks of $18 \mathrm{C} 57 \mathrm{BL} / 6 \times 129 \mathrm{~F} 1$ mice. Once tumors reached a volume of more than $1,500 \mathrm{~mm}^{3}$, mice were assigned to daily treatment (arrow) with $1 \mathrm{mg} / \mathrm{d}$ TAM i.p. plus saline (TAM/PBS) or $1 \mathrm{mg} / \mathrm{d}$ TAM i.p. plus $60 \mathrm{mg} / \mathrm{kg} / \mathrm{d}$ CQ i.p. (TAM/CQ). Results shown are daily tumor volumes (mean $\pm \mathrm{SD}$ ) for each group from a representative experiment. ${ }^{*} P<0.05$; ${ }^{* *} P<0.005$.

In the present study, CQ significantly enhanced tumor regression and delayed tumor recurrence following activation of p53 or administration of cytotoxic chemotherapy. Autophagy was activated in tumor cells that survived apoptosis following therapeutic stress. CQ treatment at low micromolar doses inhibited autophagy-dependent survival of tumor cells, and its effects were similar to that of the genetic knockdown of the essential autophagy gene ATG5 in enhancing p53-induced apoptotic cell death. Combining CQ at these doses with short hairpin RNA (shRNA) against ATG5 did not further enhance cell death following p53 activation. This study provides evidence that tumor cells can survive therapyinduced apoptosis through the process of autophagy and provides the rationale for further investigations of autophagy inhibitors as potentiators of anticancer agents.

\section{Results}

Tumor regression induced by $p 53$ is enhanced by CQ. The $p 53 E R^{T A M}$ fusion gene consists of a transcriptionally inactive hormone-binding region of the ER ( $\left.E R^{T A M}\right)$ (14) fused to the entire coding region of the Trp53 tumor suppressor gene (15). In mice homozygous for knockin alleles encoding p53ER ${ }^{\mathrm{TAM}}\left(\operatorname{Trp} 53^{\mathrm{KI} / K \mathrm{I}}\right)$, p53-dependent gene expression is induced by systemic administration of TAM (13). To generate B cell lymphomas, bone marrow cells were harvested from $\operatorname{Tr} p 53^{K I / K I}$ mice and were transduced in vivo with the LMycSN retrovirus as previously described $(16,17)$.

To examine the in vivo antitumor effect of CQ treatment in the absence of p53 activation, tumor cells were harvested from a $M y c / p 53 E R^{T A M}$ lymphoma and were injected subcutaneously into the flanks of syngeneic mice. After tumor formation, mice were matched for tumor volumes and randomly assigned to receive PBS or $60 \mathrm{mg} / \mathrm{kg} / \mathrm{d}$ CQ i.p. (Figure 1A). This dose is near the pre- viously reported $\mathrm{LD}_{50}$ of $68-78 \mathrm{mg} / \mathrm{kg}(18)$. Mice treated at these doses had no observed toxicity. Treatment with $60 \mathrm{mg} / \mathrm{kg} / \mathrm{d}$ CQ i.p. resulted in a modest but reproducible impairment in the rate of tumor growth compared with that in PBS controls. However, tumor regression was not observed in any of the CQ-treated animals. Daily treatment with the CQ derivative hydroxychloroquine (HCQ) at $60 \mathrm{mg} / \mathrm{kg} / \mathrm{d}$ resulted in similar impairment in tumor growth (data not shown).

To determine the effect of CQ treatment after therapeutic activation of apoptosis, $M y c / p 53 E R^{T A M}$ lymphomas were generated and mice were matched for tumor volume and randomly assigned to receive either daily TAM plus PBS i.p. (TAM/PBS) or daily TAM plus $60 \mathrm{mg} / \mathrm{kg} / \mathrm{d}$ CQ i.p. (TAM/CQ). TAM treatment led to nuclear localization of the $\mathrm{p} 53 \mathrm{ER}^{\mathrm{TAM}}$ fusion protein and the rapid induction of apoptosis (data not shown). TAM/PBStreated tumors regressed over several days, but all tumors resumed growth despite continued TAM therapy. TAM/CQ treatment resulted in a significant delay in tumor recurrence in comparison with TAM/PBS treatment (Figure 1B). In separate experiments, $60 \mathrm{mg} / \mathrm{kg} / \mathrm{d}$ HCQ i.p. also resulted in significantly delayed recurrence (data not shown). In all mice treated over the course of 4 separate experiments, complete clinical regression of tumor in response to therapy was observed in $81 \%$ of mice treated with TAM/CQ or TAM/HCQ compared with $8 \%$ of mice treated with TAM/PBS $(P<0.005)$.

Autophagy is activated in tumor cells that survive p53-dependent apoptosis. The activation of autophagy following oncogenic or chemotherapeutic stress has been observed in multiple cancer cell lines $(11,14)$. To further understand the effects of CQ and/or p53 activation on tumor cell autophagy in this tumor model, electron microscopy was performed on lymphoma tissue obtained from mice treated with either PBS or $60 \mathrm{mg} / \mathrm{kg} / \mathrm{d}$ CQ i.p. in the absence of p53 activation and from mice treated with either TAM/PBS or TAM/CQ at sequential time points after p53 activation. While cells that contained rare autophagosomes were found in PBS-treated tumors, tumor cells with multiple autophagosomes were easily visualized in $M y c / p 53 E R^{T A M}$ tumors treated with CQ for 96 hours in the absence of p53 activation (Figure 2A). CQ disrupts lysosomal structure and function (19), preventing effective autophagic degradation, leading to the accumulation of ineffective autophagosomes $(11,20,21)$. Eight hours after the first administration of TAM, p53 activation induced morphological changes characteristic of apoptosis in the majority of tumor cells, including chromatin condensation, nuclear and cytoplasmic blebbing, and nuclear fragmentation. By focusing on cells with intact nuclear morphology and cytoplasmic membranes, numerous tumor cells were identified in tumors from both treatment groups that had survived p53-induced apoptosis. In nonapoptotic cells, TAM/PBS treatment resulted in the appearance of large double-membrane vesicles by 24 hours and 48 hours. By 48 hours, most tumor cells had smaller residual autophagosomes as tumors began to recur. In contrast, viable tumor cells in TAM/CQ-treated tumors accumulated autophagosomes at 
A<smiles>[TeH4]</smiles>

$96 \mathrm{~h}$
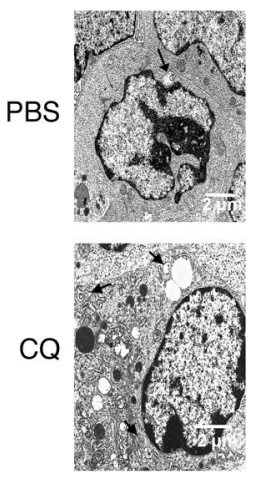

+ TAM

$24 \mathrm{~h}$
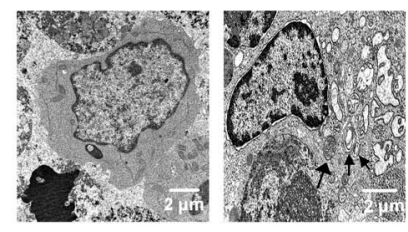

$48 \mathrm{~h}$

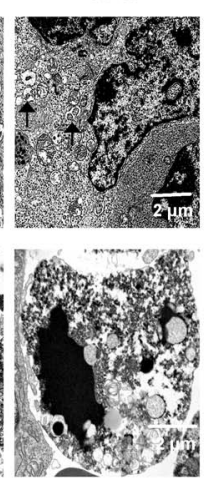

B

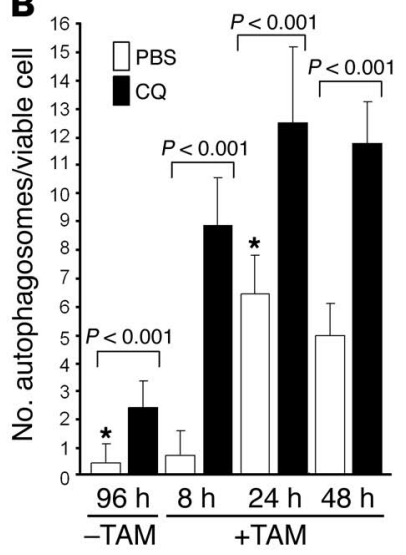

Figure 2

Effects of p53 activation with and without CQ on autophagosome accumulation. (A) Time course of changes in autophagosomes during tumor regression. Electron micrographs of lymphoma tissues collected after 96 hours of PBS or CQ treatment alone and at 8 hours, 24 hours, and 48 hours after initiation of TAM treatment. Arrows, double-membrane vesicles. Scale bars: $2 \mu \mathrm{m}$. Original magnification, $\times 10,000$. (B) Quantification of tumor cells with increased autophagosomes. Electron microscopy was performed on Myc/p53ERTAM lymphomas at the indicated time points under the treatment protocols given. The number of autophagosomes per nonapoptotic cell was determined as described in Methods (mean $\pm \mathrm{SD}$ ). ${ }^{*} P<0.005$.

8 hours following p53 activation, and by 24 hours, viable tumor cells were fewer in number and contained numerous ineffective autophagosomes with undegraded or partially degraded contents. By 48 hours, TAM/CQ-treated tumors consisted largely of the remains of apoptotic cells.

Quantification of the number of autophagosomes per nonapoptotic cell (Figure 2B) demonstrated that p53 activation alone (TAM/PBS) resulted in an 8 -fold increase in the number of autophagosomes compared with tumors treated with PBS alone by 24 hours after the initiation of TAM treatment. The appearance of numerous autophagosomes occurred earlier in TAM/CQ-treated tumors. Both in the absence of p53 activation and at each time point following p53 activation, CQ treatment resulted in a significant increase in the number of autophagosomes per nonapoptotic cell.

CQ treatment enhances $p 53$-induced apoptosis. Low magnification $(\times 4,000)$ electron micrographs of tumors treated with PBS alone compared with tumors treated with TAM/PBS or TAM/CQ at 48 hours after the initiation of TAM treatment demonstrated widespread cell death in TAM/CQ-treated tumors (Figure 3A). Morphological characteristics of apoptosis were observed in electron micrographs in $92 \% \pm 5 \%$ of tumor cells in TAM/CQ-treated tumors compared with $3 \% \pm 3 \%$ of tumor cells in TAM/PBS-treated tumors (Figure 3B). To further characterize this cell death, TUNEL staining was performed on tumor specimens to assess the number of cells undergoing apoptosis in treated tumors (Figure 3C). At 8 hours after the initiation of treatment, both TAM/PBS and TAM/CQ treatments resulted in a marked increase in TUNEL-positive tumor cells compared with PBS- and CQ-treated tumors. The number of TUNEL-positive cells decreased by 48 hours in TAM/PBStreated but not in TAM/CQ-treated tumors. Quantification of the percentage of TUNEL-positive cells per high-powered field in treated tumors (Figure 3C) found no significant differences in the percentage of TUNEL-positive cells between PBS- and CQtreated tumors and between TAM/PBS and TAM/CQ-treated tumors at 8 hours. At 24 hours, a significantly greater percentage of TUNEL-positive tumor cells was observed in TAM/CQ- treated tumors in comparison with TAM/PBS-treated tumors. This difference persisted at 48 hours, when a 7 -fold difference in the percentage of TUNEL-positive tumor cells was observed in TAM/CQ-treated tumors compared with TAM/PBS-treated tumors $(P<0.001)$. As an independent measure of tumor cell apoptosis in treated tumors, Western blot analysis of cleaved caspase-3 was performed on tumor cell lysates from TAM/PBS- and TAM/CQtreated tumors. Increased cleaved caspase-3 was observed in tumor lysates obtained at 8 hours after the initiation of either TAM/PBS or TAM/CQ. Cleaved caspase-3 was absent in TAM/PBS-treated tumor cell lysates obtained at 48 hours but present in TAM/CQtreated tumor lysates obtained at 48 hours (data not shown).

CQ enhances p53-dependent apoptosis by inhibiting autophagy. To ensure that the changes in autophagosome number seen by electron microscopy were due to the direct action of the systemically administered drugs on the autophagy pathway in tumor cells and not to changes in tumor microenvironment induced by tumor degeneration following treatment, the GFP-LC3 (LC3, mammalian homolog of yeast Atg8) fusion gene was retrovirally transduced into a bulk population of cells harvested from a primary $M y c / p 53 E R^{T A M}$ lymphoma, and GFP-positive cells were passaged in culture. LC3 is processed from LC3-I to LC3-II during autophagy. LC3-II is inserted into newly formed autophagosome membranes (22). Expression of GFP-LC3 provides a means to track changes in autophagosome formation in living cells (23). The distribution of GFP-LC3 in untreated Myc/p53ERTAM/GFP-LC3 cells was diffusely cytoplasmic (Figure 4A). CQ's ability to modulate autophagy in tumor cells was confirmed by the accumulation of LC3-positive vesicles following treatment of $M y c / p 53 E R^{T A M} / G F P-L C 3$ cells with CQ. Activation of p53 with 4-hydroxytamoxifen (hTAM) resulted in an increased number of punctate LC3-associated vesicles, which was further enhanced by combined treatment with hTAM and CQ. The accumulation of autophagosomes in CQ-treated cells was dose dependent in both the absence and presence of hTAM (Figure 4B). In the absence of hTAM, CQ doses between $500 \mathrm{nM}$ and $5 \mu \mathrm{M}$ resulted in a 2 -fold to 10 -fold increase in the percentage of cells with punctate GFP-LC3 fluorescence. Treat- 
A

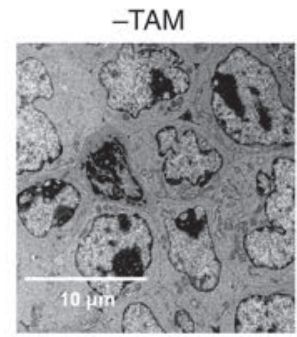

C
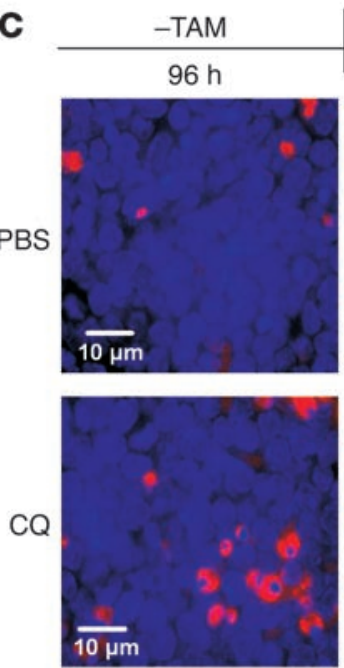

+ TAM/PBS $48 \mathrm{~h}$

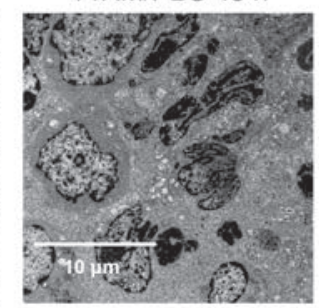

+TAM
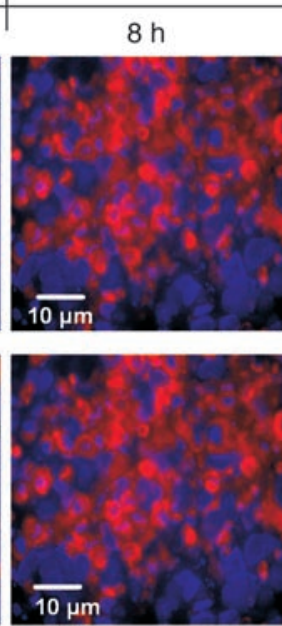

$+\mathrm{TAM} / \mathrm{CQ} 48 \mathrm{~h}$

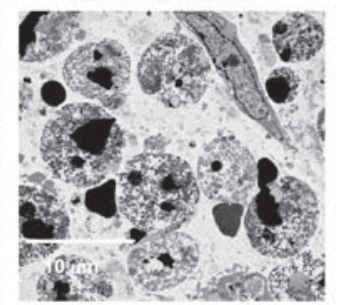

$24 \mathrm{~h}$
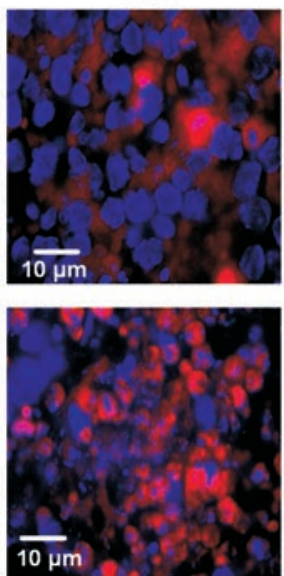
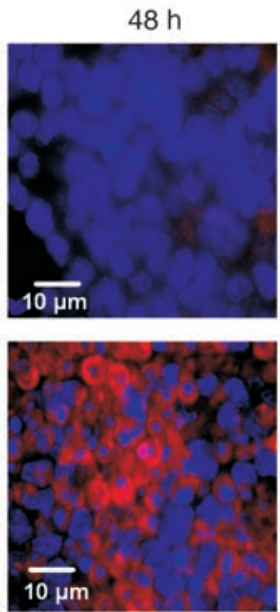

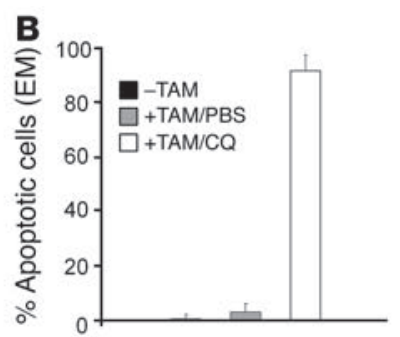

D

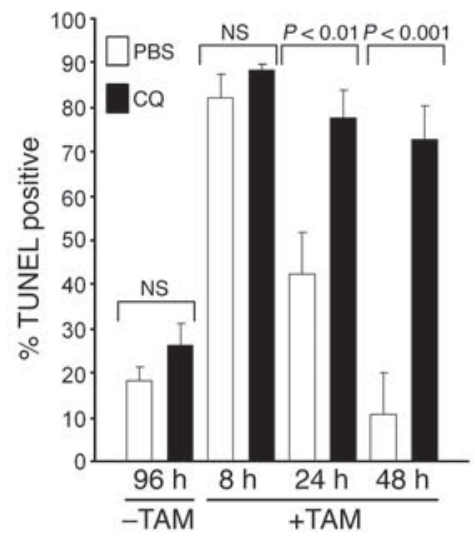

Figure 3

Effects of p53 activation with and without CQ on apoptosis. (A) CQ-induced cell death after p53 activation. Electron micrographs of lymphoma tissues collected before TAM treatment and after 48 hours of TAM/PBS or TAM/CQ. Scale bars: $10 \mu \mathrm{m}$. Original magnification, $\times 4,000$. (B) Quantification of tumor cells with morphological evidence of apoptosis. Electron microscopy (EM) was performed on Myc/p53ER ${ }^{T A M}$ lymphomas at the indicated time points under the treatment protocols given. The percentage of apoptotic cells per field at $\times 4000$ magnification was determined as described in Methods (mean \pm SD). (C) TUNEL staining was performed on tissue obtained from treated tumors at the indicated time points. Representative images were obtained by fluorescent microscopy. Red fluorescence indicates TUNEL-positive cells. Blue fluorescence indicates nuclear DAPI staining. (D) Quantification of TUNEL-positive tumor cells. The percentage of TUNEL-positive cells per high-powered field is reported as mean \pm SD.

ment with hTAM alone resulted in a 10 -fold increase in the percentage of cells with punctate GFP-LC3 fluorescence. The addition of CQ $(1-5 \mu \mathrm{M})$ during treatment with hTAM resulted in a dose-dependent increase in the percentage of cells with punctate GFP-LC3 fluorescence. These differences persisted without significant change when measured at 48 hours after treatment (data not shown). To ensure that CQ's ability to modulate autophagy was independent of $\mathrm{p} 53, p 53^{+/+}$GFP-LC3 and $p 53^{-/-}$GFP-LC3 mouse embryonic fibroblasts (MEFs) were generated. CQ induced the accumulation of punctate LC3-associated vesicles in MEFs in a $p 53$-independent manner (Figure 4C). Treatment of $p 53^{+/+} G F P-L C 3$ MEFs with hTAM did not result in punctate LC3 fluorescence (data not shown). This result confirmed that the punctate LC3 fluorescence observed in $M y c / p 53 E R^{T A M}$ lymphoma cells treated with hTAM was due to hTAM-induced activation of the p53ER ${ }^{\text {TAM }}$ fusion protein. Since the ER ${ }^{\mathrm{TAM}}$ protein domain is a transcriptionally inactivated ER, punctate GFP-LC3 fluorescence in hTAMtreated $M y c / p 53 E R^{T A M}$ cells is a p53-mediated effect.

To confirm that the antineoplastic effect of CQ observed in vivo results from the ability of CQ to inhibit autophagy-based survival, CQ treatment of tumor cells was compared with the genetic inhibition of autophagy using shRNA against the autophagy gene
ATG5 (shATG5). Expression of shATG5 blocks autophagy at a proximal step by preventing the formation of the ATG5-ATG12 complex, which is required for the generation of autophagosomes (24). shRNA designed to silence no mouse or human genes (hairpin control, HC) and 2 distinct shRNA sequences against ATG5 (shATG5 hairpin 2 [h2], shATG5 hairpin [h7]) were cloned into the control expression vector $\mathrm{pKD}(9)$ and introduced into primary tumor cells harvested from a $M y c / p 53 E R^{T A M} \mathrm{~B}$ cell lymphoma. Decreased expression levels of the ATG5-ATG12 complex in cells expressing shATG5 h2 (b2) and shATG5 h7 (b7) but not in cells expressing $\mathrm{HC}(H C)$ or vector $(V)$ was confirmed by Western blot (Figure 5A). Tumor cells were induced to undergo apoptosis when p53 was activated by hTAM treatment in vitro. p53 activation with hTAM in $b 2$ and $h 7$ cells in which ATG5 levels were chronically suppressed resulted in increased cell death compared with $H C$ cells (Figure 5B). Autophagy has been reported to promote cell survival in response to stress through both its ability to clear damaged organelles (25) and its ability to recycle intracellular contents to maintain bioenergetics (26). To determine whether the proapoptotic effect of chronic autophagy suppression could be reversed by supplying a permeable nutrient to support bioenergetics, cells were treated with hTAM and methyl pyruvate. Methyl pyruvate is a cell- 
A

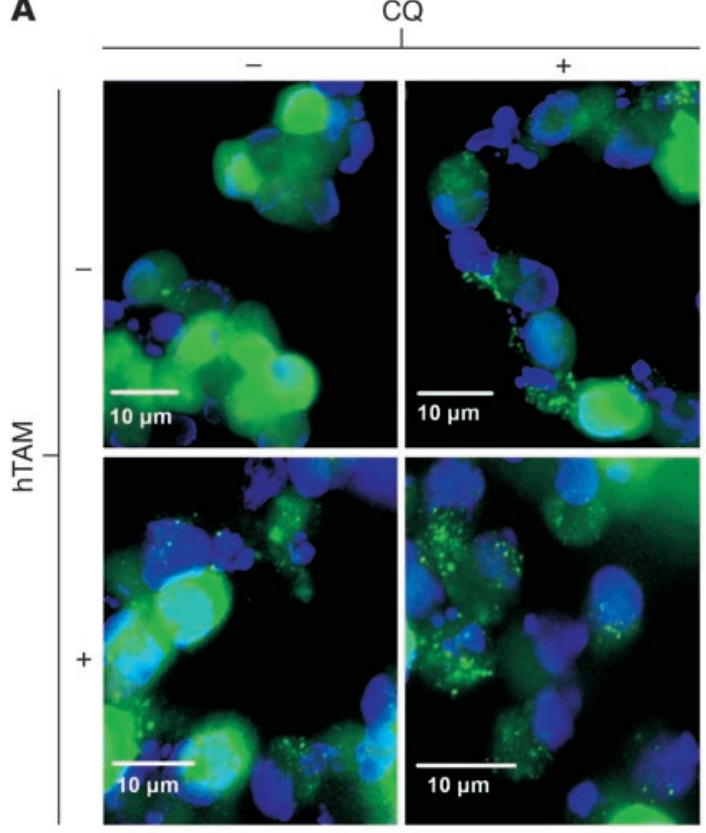

B

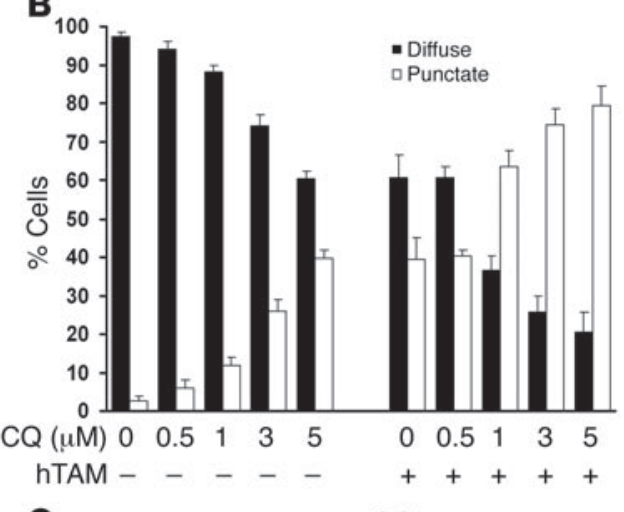

C

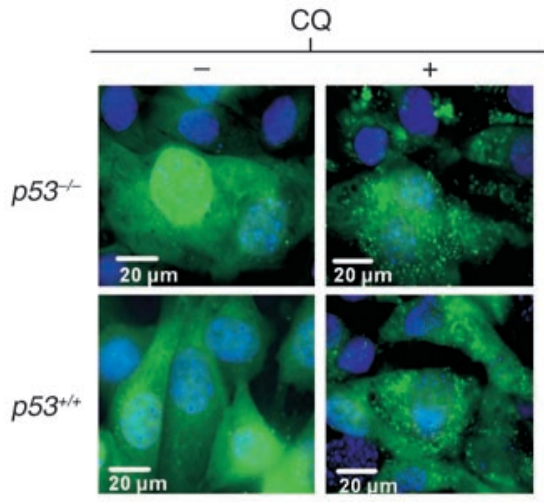

\section{Figure 4}

Effects of p53 activation with and without CQ on LC3 relocalization. (A-C) GFP-LC3 fluorescence. Green, GFP-LC3; blue, DAPI. (A) A bulk population of primary Myc/p53ER TAM lymphoma cells with stable expression of the GFP-LC3 fusion protein was treated with and without $250 \mathrm{nM}$ hTAM and with and without $5 \mu \mathrm{M} \mathrm{CQ}$. Cell culture medium was changed daily. Cells were fixed and imaged using fluorescence microscopy at 24 and 48 hours. Representative images of cells at 48 hours are presented. (B) Quantification of the percentage of cells with more than 4 GFPLC3 puncta per cell (punctate) compared with those with less than 4 GFP-LC3 puncta per cell (diffuse) treated with increasing doses of CQ with and without hTAM at 24 hours. (C) CQ modulates autophagy in a p53-independent manner. $p 53^{+/+}$and $p 53^{-/-}$MEFs expressing GFP-LC3 were treated with $C Q$. Cells were fixed and imaged at 24 hours.

permeant intermediate of glucose metabolism that has been previously reported to maintain the viability of growth factor- and/or nutrient-depleted cells in which autophagy is impaired (9). Methyl pyruvate addition to the medium failed to rescue the enhanced cell death observed in $h 2$ cells as well as $h 7$ cells following p53 activation with hTAM (Figure 5C).

The effect of CQ treatment on tumor cell death following p53 activation with hTAM was examined in cells stably transfected with $\mathrm{HC}$ or 1 of 2 distinct shATG5-expressing vectors ( $b 2, b 7$; Figure $5 \mathrm{~A}$ ). CQ treatment (1-5 $\mu \mathrm{M})$ of $H C$ cells enhanced tumor cell death in response to p53 induction in a dose-dependent fashion (Figure 6A). In this dose range, CQ alone had no reproducible effect on tumor cell viability or proliferation (Supplemental Figure 1; available online with this article; doi:10.1172/JCI28833DS1). In contrast, CQ treatment failed to enhance the cell death of either clone of shATG5-transfected cells at these doses (Figure 6, B and C).

CQ enhances tumor regression and suppresses tumor recurrence after alkylating drug therapy. In the treatment of human lymphomas, alkylating agents such as cyclophosphamide serve as first-line therapies (27-29). To determine whether the inhibition of autophagy could enhance the efficacy of alkylating drug therapy in tumors resistant to apoptosis, mice bearing $M y c / p 53 E R^{T A M}$ lymphomas were treated with cyclophosphamide alone or in combination with CQ. Mice with $M y c / p 53 E R^{T A M}$ lymphomas were treated with a single dose of $50 \mathrm{mg} / \mathrm{kg}$ cyclophosphamide i.p. followed by treatment with either PBS or $60 \mathrm{mg} / \mathrm{kg} / \mathrm{d}$ CQ i.p. The PBS or CQ treatment was then repeated daily for 13 days (Figure 7A). Cyclophosphamide with or without CQ led to complete tumor regression in all treated mice. CQ cotreatment significantly enhanced tumor regression and delayed tumor recurrence. The average tumor volume after 24 hours of treatment in cyclophosphamide/PBS-treated and cyclophosphamide/CQ-treated animals was 2,966 $\pm 673 \mathrm{~mm}^{3}$, and $1,489 \pm 524 \mathrm{~mm}^{3}$, respectively $(P<0.001)$. The addition of CQ to cyclophosphamide more than doubled the average time to recurrence of tumors. The tumors of PBS-treated mice (Figure 5) recurred after an average of $4.1 \pm 1.2$ days whereas a limited course of CQ treatment delayed tumor recurrence to an average of $9.3 \pm 3.5$ days $(P<0.01)$ (Figure 7B).

To investigate the effects of alkylating chemotherapy on autophagy, $M y c / p 53 E R^{T A M}$ cells expressing GFP-LC3 were treated with the alkylating agent $\mathrm{N}$-methyl-N'-nitro-N-nitrosoguanidine (MNNG) with and without CQ (Figure 7C). MNNG is an alkylating agent that can induce apoptosis in a p53-independent manner (30). CQ treatment or MNNG treatment alone resulted in an increased percentage of viable cells with punctate LC3 fluorescence, indicating an accumulation of autophagosomes. Combination treat- 
A

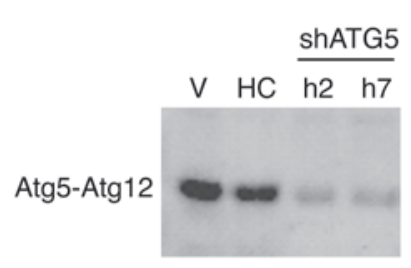

Actin

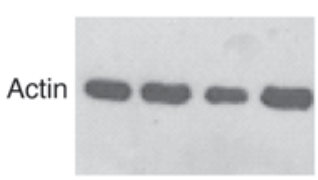

\section{B}

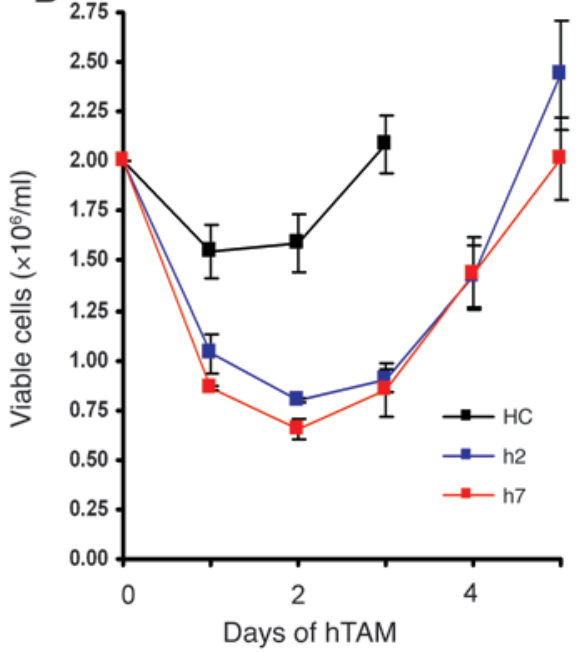

C

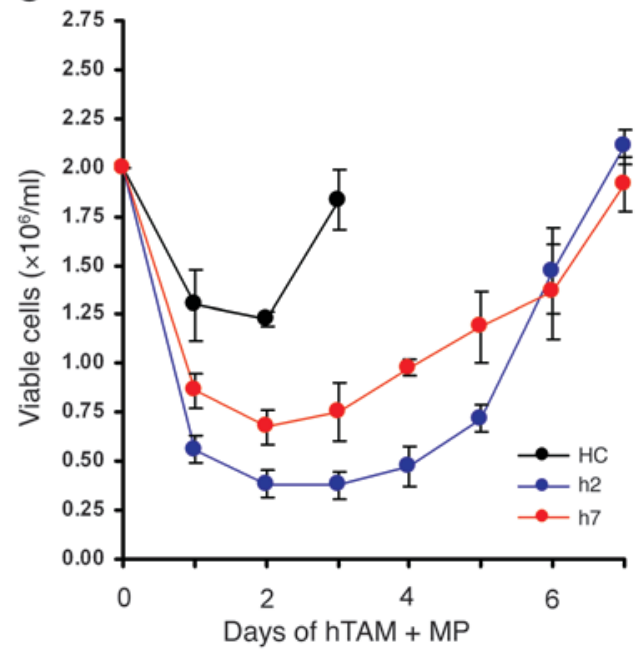

Figure 5

Effects of p53 activation with and without knockdown of ATG5 on tumor cell death. (A) Western blot against ATG5 of lysates from Myc/p53ERTAM/ vector cells (V), Myc/p53ERTAM/HC cells (HC), Myc/p53ERTAM/shATG5 h2 cells (h2), and Myc/p53ERTAM/shATG5 h7 cells (h7). Actin was used as a loading control. (B) Activation of p53 in HC and shATG5 cells. HC, $h 2$, and $h 7$ lymphoma cells were treated with $200 \mathrm{nM}$ hTAM daily. (C) Activation of p53 plus methyl pyruvate (MP). HC, $h 2$, and $h 7$ lymphoma cells were treated daily with hTAM plus $1 \mathrm{mM}$ MP. (B and C) Viable cell number as determined by trypan blue exclusion was counted daily. Reported values are mean \pm SD of triplicate samples from a representative experiment.

ment with MNNG and CQ resulted in 9-fold and 17-fold increases in punctate LC3 fluorescence compared with DMSO control at 24- and 48-hour time points (Figure 7D). To further compare the effect of genetic autophagy inhibition with that of CQ following cytotoxic therapy, the effect of MNNG treatment with and without CQ was tested in $H C, h 2$, and $h 7$ cells. At 24 and 48 hours, combined treatment with $5 \mu \mathrm{M}$ CQ significantly enhanced the cytotoxic effect of MNNG treatment of $H C$ lymphoma cells to a degree similar to that of treatment of $h 2$ or $b 7$ cells with MNNG alone. CQ treatment did not further enhance cell death induced by an alkylating agent in $b 2$ or $h 7$ cells (Figure 7E).

\section{Discussion}

The results described in this study provide evidence that autophagy can be an adaptive mechanism that contributes to tumor cell survival and resistance to therapy-induced apoptosis. Induction of p53 in Mycp53ER ${ }^{T A M}$ tumors results in the induction of tumor cell apoptosis, and tumor cells that survive the acute induction of p53-induced apoptosis display active autophagy. Impairment of autophagic vesicle clearance by the lysosomotropic drug CQ correlated with enhanced apoptosis and tumor regression as well as delayed tumor recurrence. This appears to be due to a direct prosurvival effect of autophagy in tumor cells, since autophagy inhibition by either ATG5 shRNA or CQ enhances tumor cell apoptosis and suppresses tumor cell recovery when p53 is induced in vitro.

CQ has the ability to disrupt lysosomal function (10), inhibiting the last critical step in autophagy, the acid-dependent degradation of autophagosome contents, which results in the accumulation of autophagic vesicles that cannot be cleared (11). Although CQ at higher doses may have additional detrimental effects $(31,32)$ on tumor cell viability, the CQ doses studied here failed to cause tumor regression in vivo or induce the death of tumor cells in vitro. The ability of CQ to augment tumor cell death in response to p53 activation or alkylating drug therapy correlated with its ability to impair autophagy. A similar augmentation of tumor cell death in response to 553 activation or alkylating drug therapy was observed in cells in which autophagy is genetically suppressed by shATG5. CQ treatment failed to further augment cell death in cells lacking functional autophagy following p53 activation or alkylating drug therapy. Thus, at the doses studied here, inhibition of autophagy is likely the basis of CQ's major antineoplastic effect. Together, these data demonstrate that inhibitors of autophagy enhance the efficacy of therapeutic strategies designed to induce tumor cell apoptosis.

Prior studies have led to conflicting views of the role of autophagy in tumor cell biology. Suppression or deficiency of autophagy genes has been shown to enhance tumorigenesis (6), leading to the conclusion that rapidly growing tumors downregulate autophagy. Consistent with this finding, the autophagy-associated tumor suppressor gene BECN1 is monoallelically deleted in many breast cancers, leading to reduced autophagy in the tumor cells $(33,34)$. These observations suggest that autophagy may suppress tumor cell outgrowth and raise the possibility that pharmacologic suppression of autophagy might enhance tumor cell growth and survival. In contrast, recent work has suggested that autophagy plays an important role in mammalian cell biology by providing cells an adaptive mechanism to survive bioenergetic stress as a result of either growth factor or nutrient deprivation (9). A similar tumorprotective role for autophagy was identified by other investigators using an Akt-driven xenograft model defective in apoptosis. In this model, autophagy was activated in tumor cells at the center of growing tumors and served to protect tumor cells from necrosis induced by the combination of an activated oncogene and nutrient-limited conditions typically encountered by tumor cells in vivo. Interestingly, to date no tumors have been reported in which biallelic inactivation of BECN1 or another autophagy gene has been observed, suggesting that the preservation of autophagy as an inducible survival mechanism is required for tumorigenesis. Selection for monoallelic loss of autophagy genes during tumori- 
A

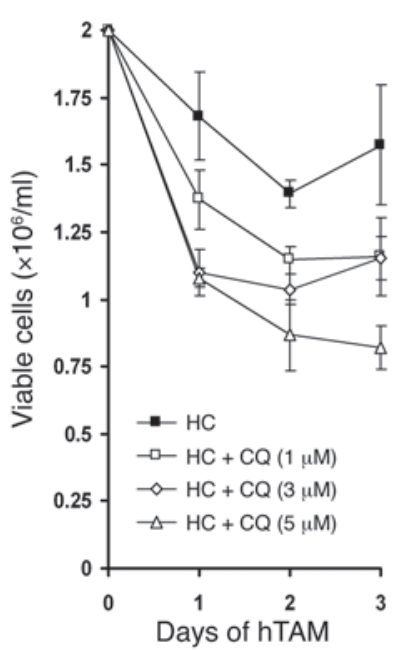

B

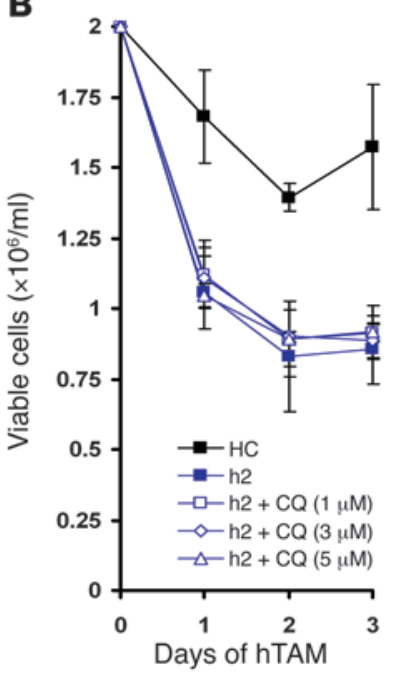

C

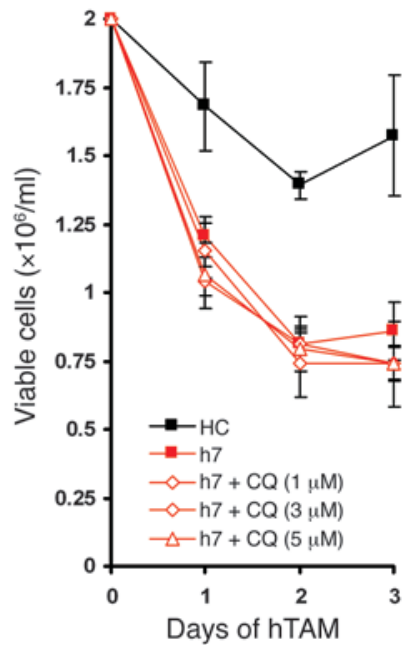

Figure 6

Effects of CQ or ATG5 knockdown in combination with p53 activation on tumor cell death. (A) Activation of p53 plus CQ in $H C$ cells. $H C$ lymphoma cells were treated daily with $200 \mathrm{nM}$ hTAM plus $1 \mu \mathrm{M}, 3 \mu \mathrm{M}$, or $5 \mu \mathrm{M} \mathrm{CQ}$. (B) Activation of p53 plus CQ in $h 2$ lymphoma cells. HC cells were treated daily with hTAM and compared with $h 2$ cells treated daily with hTAM or hTAM plus $1 \mu \mathrm{M}, 3 \mu \mathrm{M}$, or $5 \mu \mathrm{M}$ CQ. (C) Activation of p53 plus CQ in $h 7$ lymphoma cells. HC cells were treated daily with hTAM and compared with $h 7$ cells treated daily with hTAM or hTAM plus $1 \mu \mathrm{M}$, $3 \mu \mathrm{M}$, or $5 \mu \mathrm{M} \mathrm{CQ}$. (A-C) Viable cell number as determined by trypan blue exclusion was counted daily. Reported values are mean \pm SD of triplicate samples from a representative experiment.

genesis may be related to the reported function of autophagy in eliminating damaged or excess organelles $(35,36)$. Yeast defective in UTH1, which encodes a mitochondrial protein required for effective targeting of mitochondria for autophagic degradation (37), are hypersensitive to certain types of oxidant injury (38). Therefore, chronic suppression of autophagy over a long period of time would result in the accumulation of cellular oxidants that damage DNA, increasing the likelihood of cellular transformation. The role of autophagy in suppressing the accumulation of oxidative damage to cells may account for autophagy's role in suppressing tumorigenesis while serving a tumor-protective effect against various types of cellular stress encountered by established tumors.

The present studies were undertaken because of numerous reports of autophagy observed in established cancer cell lines following anticancer therapy $(1-3,39)$. The data presented above demonstrate the induction of autophagy in tumor cells in vivo in response to the activation of $\mathrm{p} 53$, a gene commonly induced by a number of antineoplastic therapies. It has been suggested that the ability of radiation or chemotherapy to induce cell death in cancer cell lines that display resistance to apoptosis depends on type II programmed cell death executed by autophagy (40). In Myc-induced lymphoma cells, knockdown of ATG5, an essential autophagy gene, did not impair p53-induced cell death, suggesting that the autophagy program that is activated with apoptosis does not contribute to cell death. Instead, autophagy is serving a survival function in this context since expression of shRNA directed against ATG5 in lymphoma cells augmented cell death following p53 activation. These data indicate that autophagy can be an adaptive response that allows cancer cells to survive an apoptotic stimulus that would otherwise lead to their demise.

The ability of tumor cells expressing ATG5 shRNA to grow suggests that once neoplastic proliferation is established, autophagy is not absolutely required for in vitro cell growth and survival.
However, our data suggest that there is ongoing autophagy during $M y c / p 53 E R^{T A M}$ tumor growth, based on the accumulation of autophagic vesicles when their clearance by lysosomes is inhibited by CQ. When tumor cells are faced with cellular stress that induces apoptosis, autophagy serves to protect against cell death. Inhibition of autophagy in the setting of an apoptotic stress enhances apoptosis. Since autophagy contributes to cell survival in times of stress, it seems likely it can provide enhanced survival to tumor cells subjected to potentially catastrophic stress even when expressed in a haploinsufficient manner.

The tumors and tumor cells used in this study to investigate the role of autophagy following apoptotic stress were created by expressing wild-type human $c-M y c$ in mouse bone marrow cells from the p53ER-knockin mouse. In addition to Burkitt lymphoma, in which a chromosomal translocation juxtaposes the $c-M y c$ gene to 1 of 3 immunoglobulin genes, leading to its constitutive expression in B cells (41), dysregulation of $c-M y c$ through gene amplification (42-44), c-Myc point mutations (45), or constitutive transcriptional and posttranslational activation $(46,47)$ has been implicated in the pathogenesis of multiple malignancies, including lung cancer, colon cancer, and breast cancer. The role of Myc as a transcriptional regulator of genes that affect glucose metabolism and its propensity to induce apoptosis (48) when expressed acutely in nontransformed cells raises the possibility that autophagy observed in these tumors may be a specific consequence of introducing a therapeutic stress on top of $M y c$-induced cellular stress. Recently, however, autophagy was found to promote tumor survival in another tumor model driven by the oncogene Akt (49). Akt, when activated, also increases glycolytic metabolism and controls apoptosis. In these tumors, autophagy is activated and preferentially protects tumor cells in the centers of growing Aktdriven, apoptosis-deficient tumors, where nutrients and oxygen are limited. This suggests that oncogenes that activate the War- 
A

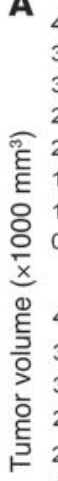

4.0 CY

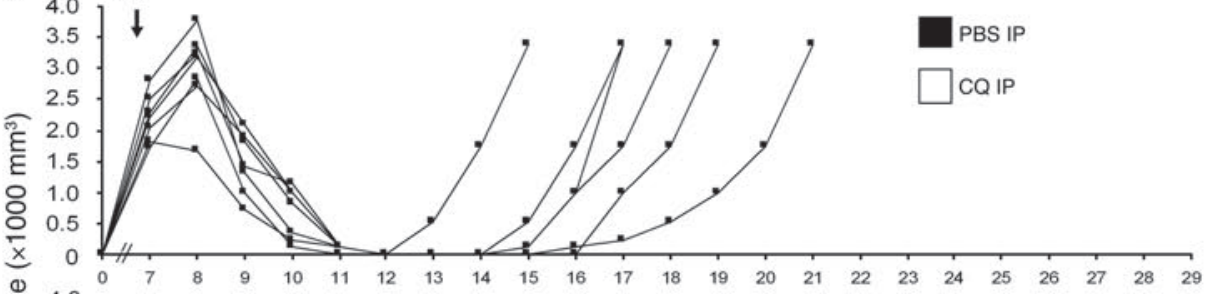

를 4.0
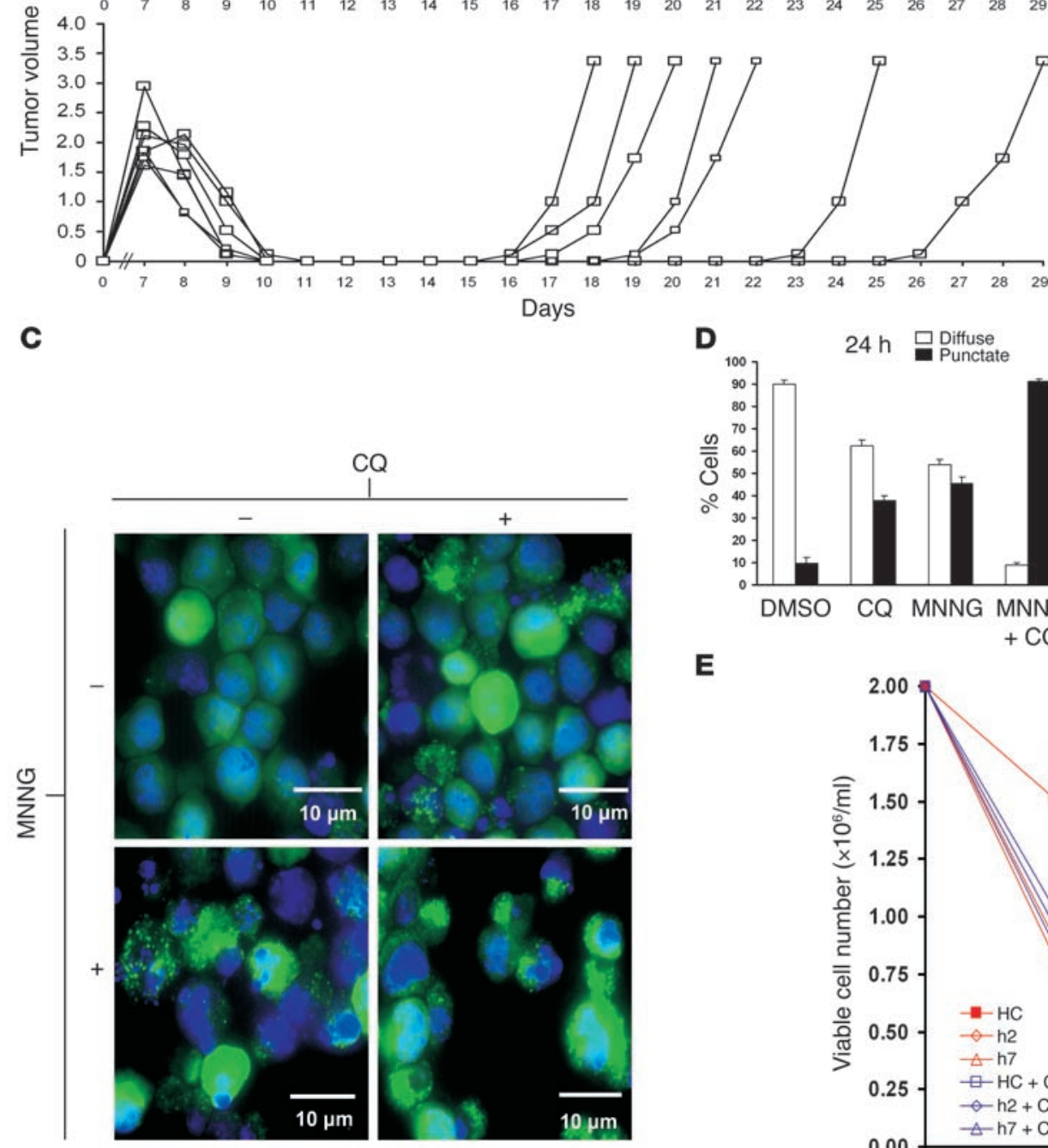

Days

\section{B}

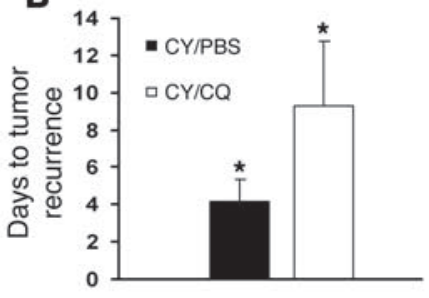

C

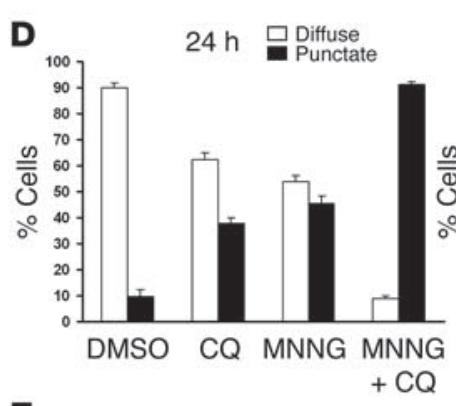

E

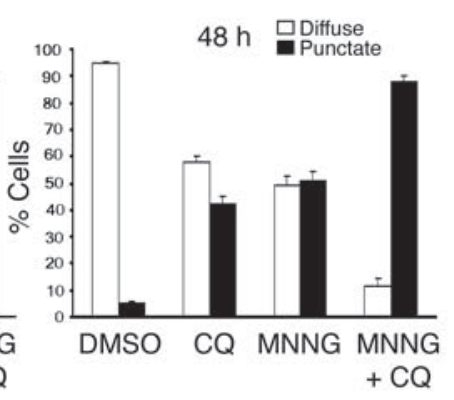

\section{Figure 7}

Effects of alkylating chemotherapy with and without CQ in Myc/p53ER TAM lymphomas. (A) Cells from a primary tumor were harvested and passaged in vivo in syngeneic C57BL/6×129F1 mice. Myc/p53ER TAM cells were injected subcutaneously into the flanks of mice. Once tumors had reached more than $1,700 \mathrm{~mm}^{3}$, mice were matched for tumor size and treated with $50 \mathrm{mg} / \mathrm{kg}$ cyclophosphamide i.p. once followed by either daily PBS i.p. (top panel) or $60 \mathrm{mg} / \mathrm{kg} / \mathrm{d} \mathrm{CQ}$ i.p. (bottom panel) for a total of 13 days. Daily tumor volumes are shown for individual mice. $\mathrm{CY}$, cyclophosphamide. (B) Time to tumor recurrence for cyclophosphamide/PBS- and cyclophosphamide/CQ-treated mice (mean \pm SD). ${ }^{*} P=0.003$. (C) GFP-LC3 fluorescence. Green, GFP-LC3; blue, DAPI. A bulk population of primary Myc/p53ER TAM lymphoma cells with stable expression of the GFP-LC3 fusion protein was treated with $50 \mu \mathrm{M}$ MNNG with or without $10 \mu \mathrm{M} \mathrm{CQ}$. Cell culture medium was changed daily. Cells were fixed and imaged using fluorescence microscopy at 24 and 48 hours. Representative images of cells at 48 hours are presented. (D) Quantification of the percentage of cells with more than 4 GFP-LC3 puncta per cell (punctate) compared with those with less than 4 GFP-LC3 puncta per cell (diffuse) at 24 and 48 hours after treatment. (E) MNNG with and without CQ treatment in $H C$ and shATG5 cells. On day $0,2 \times 10^{6}$ cells $/ \mathrm{ml}$ of $H C, h 2$, and $h 7$ lymphoma cells were plated and treated with $20 \mu \mathrm{M}$ MNNG (red) or $20 \mu \mathrm{M}$ MNNG plus $5 \mu \mathrm{M}$ CQ (blue). Viable cell number as determined by trypan blue exclusion was counted after 24 hours and 48 hours of treatment. Reported values are means \pm SD of triplicate samples of a representative experiment. 
burg effect, such as Akt, and drive the glycolytic phenotype frequently seen in a variety of tumors may predispose tumor cells to increase autophagy as a strategy for survival in the face of nutrient limitation. However, in $M y c / p 53 E R^{T A M}$ cells, the permeable nutrient methyl pyruvate did not rescue the enhanced p53-induced apoptosis observed in tumor cells in which autophagy was genetically silenced. Autophagy can promote cell survival not only by recycling cellular constituents to support bioenergetics but also by clearing damaged organelles, whose accumulation following cellular stress can further damage cells. Autophagy induced in response to $\mathrm{p} 53$-induced stress and cytotoxic chemotherapy may provide the tumor cell a means of clearing damaged organelles. Together with evidence of the role of autophagy as a tumor survival pathway in Akt-driven tumors, the data presented in this study of Mycdriven lymphomas suggest that autophagy may serve as a generalized survival function in oncogene-transformed cells. Further investigation is required to fully understand the contribution of other oncogenes and tumor suppressor genes in determining the outcome of combined autophagy inhibition and apoptosis induction. Nevertheless, many of the existing and experimental chemotherapeutic approaches for the treatment of cancer seek to induce tumor cell apoptosis. The data presented here demonstrate that autophagy in response to either p53 activation or alkylating drug therapy can contribute to the tumor cell's ability to resist apoptosis. These studies identify CQ and the related compound HCQ as inhibitors of autophagy that can be used in vivo.

The current data suggest that CQ may be an important adjunct to enhance the efficacy of existing chemotherapeutic strategies without potentiating toxicity. CQ cotreatment with TAM or with the alkylating agent cyclophosphamide did not result in additional toxicity in the treated animals. This is consistent with the fact that CQ has been used safely for decades in patients for malaria prophylaxis (50) and for the treatment of rheumatoid arthritis, where it is often used in combination with methotrexate (51). The chemical structure of CQ derivatives allows them to serve as weak bases that become trapped in acidic compartments. Since glycolytic tumors are characteristically more acidic than surrounding normal tissue (52), CQ derivatives may preferentially accumulate in tumor tissue and display greater efficacy in the inhibition of autophagy in tumor versus normal tissue. Systemic administration of CQ at doses roughly equivalent to human doses used to treat malaria or rheumatoid arthritis was well tolerated for up to 20 days in mice. Although CQ has been reported to have a variety of additional cellular effects in addition to its ability to suppress autophagy, the ability of low doses of CQ to enhance p53-induced apoptosis was dependent on its effects on autophagy. While ATG5 shRNA independently enhanced p53-induced cell death, no further enhancement of cell death was observed when tumor cells expressing ATG5 shRNA were treated with low doses of CQ. However, at high doses (10 $\mu \mathrm{M}$ or greater), CQ treatment may have autophagy-independent effects (Supplemental Figure 1).

Although CQ derivatives such as HCQ at maximally tolerated doses may achieve a peak blood concentration approaching $10 \mu \mathrm{M}$, numerous pharmacokinetic studies of HCQ as a single agent at the standard doses used in rheumatoid arthritis have determined that steady-state blood concentrations are in the range of $2-3 \mu \mathrm{M}(53,54)$. Together, these data provide the rationale for further investigation of combination regimens including CQ derivatives and systemic chemotherapy and/or radiation in order to enhance the therapeutic efficacy of existing cancer therapies. The data suggest that the development of more specific autophagy inhibitors may be of clinical benefit if they can be utilized in combination with apoptosis-inducing agents.

\section{Methods}

Tumorgeneration and tissue isolation. All experiments were performed in accordance with approved animal safety protocols. Approval for animal care and use for these experiments was provided by the Institutional Animal Care and Use Committee (IACUC) at the University of Pennsylvania. p53ER TAM/ p53ER ${ }^{T A M}$ mice were created by M.A. Christophorou, D. Martin-Zanca (BRI-Basic Research Program, National Cancer Institute-Frederick Cancer Research Facility, Bethesda, Maryland, USA), and G.I. Evan. All experiments were carried out using 8- to 10 -week-old C57BL/6×129F1 mice obtained from The Jackson Laboratory. Bone marrow cell harvest and production of bone marrow-derived neoplasms followed the protocol previously described (16). After primary tumor formation, tumor cells were harvested in ice-cold PBS by passage through a 70- $\mu \mathrm{m}$ nylon mesh (BD Biosciences) and expanded in vivo by subcutaneous injection into the flanks of syngeneic mice. For tissue analysis, all animals were sacrificed individually by $\mathrm{CO}_{2}$ asphyxiation and tissue was harvested immediately. Tumors were harvested in ice-cold PBS. For each tumor, sections of visually viable tumor tissue were fixed in $10 \%$ formalin for preparation of paraffin-embedded sections and glutaraldehyde for electron microscopy (see below). Tumor cell lysates were achieved through manual agitation of remaining tumor tissue in RIPA buffer.

Drug administration and tumor measurements. For TAM treatment, the hormone powder (Sigma-Aldrich) was dispersed, via sonication, in peanut oil (Sigma-Aldrich) at a concentration of $10 \mathrm{mg} / \mathrm{ml}$. The administration of TAM was done via daily i.p. injections at the dose of $1 \mathrm{mg}$ per mouse. CQ (Sigma-Aldrich) and HCQ (Spectrum Chemicals Ltd.) were both dissolved in PBS and administered i.p. For in vitro studies, CQ was dissolved in PBS. hTAM (Sigma-Aldrich) was dissolved in ethanol. Cyclophosphamide (Sigma-Aldrich) was dissolved in PBS. MNNG (Sigma-Aldrich) was dissolved in DMSO. Methyl pyruvate was diluted in PBS (Sigma-Aldrich). For experiments involving MNNG treatment of cells in vitro, cells were resuspended in RPMI containing 10\% FBS with indicated drugs and incubated at $37^{\circ} \mathrm{C}$ for 15 minutes. Cells were spun down and resuspended in fresh media with LPS and IL-7 at appropriate doses \pm CQ as indicated. Cells were re-treated in this manner daily. Tumors were measured on a daily basis using calipers, and tumor volume was calculated using the following formula: $\left(\mathrm{mm}^{3}\right)=A \times B \times[(A+B) / 2] . A$ and $B$ were the largest measurements of length and width, respectively, for each tumor.

Cell culture. For in vitro experiments, 1 primary $M y c / p 53 E R^{T A M}$ tumor was harvested in cold PBS, and tumor cells were strained through a $70-\mu \mathrm{m}$ nylon mesh (BD Biosciences) to isolate a bulk population of tumor cells. Cells were then frozen in aliquots for future experiments. All in vitro experiments were done in complete medium consisting of RPMI 1640 medium (Invitrogen) supplemented with 10\% heat-inactivated FBS (Gemini Bio-Products), $10 \mathrm{U} / \mathrm{ml}$ penicillin/streptomycin, and $2 \mathrm{mM}$ L-glutamine (Invitrogen). Also, $10 \mu \mathrm{g} / \mathrm{ml}$ lipopolysaccharide (Sigma-Aldrich) and $0.2 \mathrm{ng} / \mathrm{ml}$ IL-7 (R\&D Systems) were added daily. For all in vitro experiments, media plus supplements and drug treatments were changed daily. Cell number was assessed by using a Coulter Z2 particle analyzer or trypan blue exclusion. For MTS assays, $2 \times 10^{5}$ cells/well were plated in 96-well plates in RPMI medium containing IL-7, LPS, and the indicated drug treatments. At 24 hours, the MTS reagent supplied in CellTiter 96 AQueous Assay (Promega) was added and the results were analyzed according to the manufacturer's recommendations. The $p 53^{+/+}, p 53^{-/-}, p 53^{+/+} G F P-L C 3$, and $p 53^{-/-G F P-L C 3}$ MEF cell lines were passaged in DMEM medium supplemented with $10 \%$ heat-inactivated FBS (Gemini Bio-Products), $10 \mathrm{U} / \mathrm{ml}$ penicillin/streptomycin, and $2 \mathrm{mM} \mathrm{L-glutamine} \mathrm{(Invitrogen).}$ 
Immunoblotting. Cultured cells were lysed in RIPA buffer. Tumor lysates were obtained by manual agitation of tumor tissue and lysis in RIPA. Lysates were standardized for protein content and resolved by SDS-PAGE on 14\% NuPAGE gels (Invitrogen). Nitrocellulose blots were probed with antibodies against cleaved caspase-3 (rabbit monoclonal antibody, 1:000; Cell Signaling Technology); anti-actin (mouse monoclonal antibody, 1:10,000; Sigma-Aldrich); and anti-ATG5 (rabbit polyclonal antibody, 1:2,000; gift from N. Mizushima, Tokyo Medical and Dental University, Tokyo, Japan).

Electron microscopy quantification of autophagosomes and apoptosis. Tissue obtained from tumors was immediately fixed with $2.5 \%$ glutaraldehyde $/ 2 \%$ formaldehyde with $0.1 \mathrm{M}$ sodium cacodylate and stored at $4{ }^{\circ} \mathrm{C}$ until embedding. Cells were postfixed with $2 \%$ osmium tetroxide; this was followed by an increasing gradient dehydration step using ethanol and propylene oxide. Cells were then embedded in LX-112 medium (Ladd), and sections were cut ultrathin $(90 \mathrm{~nm})$, placed on uncoated copper grids, and stained with $0.2 \%$ lead citrate and $1 \%$ uranyl acetate. Images were examined with a JEOL-1010 electron microscope (JEOL) at $80 \mathrm{kV}$. For quantification of viable cells using electron micrographs of tumor tissue, high-powered micrographs $(\times 12,000-20,000)$ of 25 single cells from multiple distinct low-powered fields in each tumor were obtained. Cells were considered nonapoptotic if the integrity of the nuclear and cytoplasmic membrane was maintained. For quantification of apoptotic cells, cells with cytoplasmic and nuclear blebbing and condensed chromatin or apoptotic bodies were scored as apoptotic. For quantification of autophagic vesicles per viable cell, the number of double-membrane vesicles per nonapoptotic cell was scored. Data are presented as mean \pm SD.

TUNEL staining and fluorescence imaging. TUNEL staining was performed using the In Situ Cell Death Detection Kit, TMR Red (Roche Diagnostics) on paraffin-embedded tissue harvested from tumors per manufacturer's instructions. DAPI counterstain was used to quantify cells with intact nuclei. The percentage of TUNEL-positive cells was calculated by dividing the number of TUNEL-positive cells by the number of DAPI-positive nuclei at $\times 100$ magnification for 4 fields for each tumor sampled. For GFP-LC3 fluorescence imaging, Myc/p53ER ${ }^{\text {TAM }} /$ GFP-LC3, $p 53^{+/+} G F P-L C 3$, and $p 53^{--}$GFP-LC3 (see below) cells were exposed to the indicated treatments and fixed with $4 \%$ paraformaldehyde for 30 minutes at room temperature, washed 3 times, and centrifuged onto slides. DAPI counterstain was used to identify cells with intact nuclei. All fluorescence imaging was performed and digitally captured at $\times 100$ magnification on a Nikon Eclipse E800 fluorescent microscope.

Constructs, retroviral infection, and RNA interference. The U6 promoter and hairpin sequence of the shRNA nontarget vector pLKO-puro (SigmaAldrich) was cloned into TOPO pCR 2.1 (Invitrogen), and the BamHI/ SnaBI fragment was excised and cloned into the $\mathrm{pKD}$ expression vector to create $\mathrm{HC}$ vector. Two distinct shRNA sequences against ATG5 (shATG5 h2, shATG5 h7) were generated and cloned into the pKD expression vec- tor (constructed from pBABE-GFP) as previously described (9). The plasmid MIGR1/GFP-LC3 was constructed by cloning an XhoI site $5^{\prime}$ to the GFP-LC3 coding sequence of the PEGFP-C1/LC3 vector (generous gift of T. Yoshimori, CREST, Japan Science and Technology Agency, KawaguchiSaitama, Japan). The XhoI/EcoRI fragment containing the coding region of the GFP-LC3 fusion gene was inserted into the multiple cloning site of MIGR1 to generate the MIGR1/GFP-LC3 plasmid. For production of hightiter retrovirus, $293 \mathrm{~T}$ cells were cotransfected with retroviral vector $(5 \mu \mathrm{g})$ plus helper DNA $(2.5 \mu \mathrm{g})$ using Lipofectamine 2000 (Invitrogen). The following retroviral vectors were used: MIGR1/GFP-LC3, $\mathrm{pKD}, \mathrm{pKD} / \mathrm{HC}$, $\mathrm{pKD} /$ shATG5 h2, and pKD/shATG5 h7. Conditioned media was harvested and filtered through a $0.45-\mu \mathrm{M}$ filter. Culture supernatants were then used to transduce Myc/p53ER ${ }^{\text {TAM }}$ cells, $p 53^{+/+}$MEFs, and $p 53^{-/-}$MEFs. Next, $2 \times 10^{6}$ cells were plated in $1 \mathrm{ml}$ of conditioned media containing MIGR1/GFPLC3, pKD, pKD/HC, pKD/shATG5 h2, or pKD/shATG5 h7 in the presence of $8 \mu \mathrm{g} / \mathrm{ml}$ hexadimethrine bromide (Polybrene; Sigma-Aldrich). For $M y c / p 53 E R^{T A M}$ cells, fresh IL-7 and LPS were added at the indicated concentrations in a 24-well plate. Adherent cells were infected and incubated for 4-5 hours. For suspension cells, culture plates were spun at $1,015 \mathrm{~g}$ for 1 hour at room temperature and then incubated at $37^{\circ} \mathrm{C}$ for 2 hours. Infection was repeated in this fashion 3 times. Cells were then resuspended in the appropriate medium and expanded in culture for 3 days. Transduced cells were sorted for GFP-positive cells (MoFlo; Cytomation) and further cultured.

Statistics. Means were compared using the 2-tailed Student's $t$ test. $P<0.05$ was considered statistically significant in all calculations. All data analyses were performed using GraphPad QuickCalcs version 1.

\section{Acknowledgments}

The authors would like to thank Neelima Shah for technical assistance with electron microscopy and Qian-Chun Yu, Hongwei $\mathrm{Yu}$, and Yan Wang for technical assistance with tissue processing. We also would like to thank members of the Thompson Laboratory, especially Roland Knoblauch, Russell Jones, Monica Buzzai, and Fangping Zhao, for helpful discussions. Grant support was provided by the NIH (R25-CA87812 to R.K. Amaravadi; R01-CA 102709 to D. Yu and A. Thomas-Tikhonenko; and R01CA104838-01A1 to T. Bui and C.B. Thompson) and the Leukemia and Lymphoma Society (to J.J. Lum).

Received for publication April 17, 2006, and accepted in revised form November 28, 2006.

Address correspondence to: Craig B. Thompson, Department of Cancer Biology, Abramson Family Cancer Research Institute, 421 Curie Boulevard, 450 BRB II/III, Philadelphia, Pennsylvania 19104, USA. Phone: (215) 746-5515; Fax: (215) 746-5511; E-mail: craig@ mail.med.upenn.edu.
1. Kanzawa, T., et al. 2004. Role of autophagy in temozolomide-induced cytotoxicity for malignant glioma cells. Cell Death Differ. 11:448-457.

2. Paglin, S., et al. 2001. A novel response of cancer cells to radiation involves autophagy and formation of acidic vesicles. Cancer Res. 61:439-444.

3. Bursch, W., et al. 1996. Active cell death induced by the anti-estrogens tamoxifen and ICI 164384 in human mammary carcinoma cells (MCF-7) in culture: the role of autophagy. Carcinogenesis. 17:1595-1607.

4. Kondo, Y., Kanzawa, T., Sawaya, R., and Kondo, S. 2005. The role of autophagy in cancer development and response to therapy. Nat. Rev. Cancer. 5:726-734.

5. Yu, L., et al. 2004. Regulation of an ATG7-beclin 1 program of autophagic cell death by caspase-8. Science. 304:1500-1502.

6. Qu, X., et al. 2003. Promotion of tumorigenesis by heterozygous disruption of the beclin 1 autophagy gene. J. Clin. Invest. 112:1809-1820. doi:10.1172/ JCI200320039.

7. Kiffin, R., Christian, C., Knecht, E., and Cuervo, A.M. 2004. Activation of chaperone-mediated autophagy during oxidative stress. Mol. Biol. Cell. 15:4829-4840

8. Benhar, M., Dalyot, I., Engelberg, D., and Levitzki, A. 2001. Enhanced ROS production in oncogenically transformed cells potentiates c-Jun N-terminal kinase and p38 mitogen-activated protein kinase activation and sensitization to genotoxic stress. Mol. Cell. Biol. 21:6913-6926.
9. Lum, J.J., et al. 2005. Growth factor regulation of autophagy and cell survival in the absence of apoptosis. Cell. 120:237-248.

10. Poole, B., and Ohkuma, S. 1981. Effect of weak bases on the intralysosomal $\mathrm{pH}$ in mouse peritoneal macrophages. J. Cell Biol. 90:665-669.

11. Glaumann, H., and Ahlberg, J. 1987. Comparison of different autophagic vacuoles with regard to ultrastructure, enzymatic composition, and degradation capacity--formation of crinosomes. Exp. Mol. Pathol. 47:346-362.

12. Gonzalez-Polo, R.A., et al. 2005. The apoptosis/ autophagy paradox: autophagic vacuolization before apoptotic death. J. Cell Sci. 118:3091-3102.

13. Christophorou, M.A., et al. 2005. Temporal dissection of $\mathrm{p} 53$ function in vitro and in vivo. Nat. Genet. 
37:718-726.

14. Littlewood, T.D., Hancock, D.C., Danielian, P.S., Parker, M.G., and Evan, G.I. 1995. A modified oestrogen receptor ligand-binding domain as an improved switch for the regulation of heterologous proteins. Nucleic Acids Res. 23:1686-1690

15. Vater, C.A., Bartle, L.M., Dionne, C.A., Littlewood, T.D., and Goldmacher, V.S. 1996. Induction of apoptosis by tamoxifen-activation of a p53-estrogen receptor fusion protein expressed in E1A and T24 H-ras transformed p53-/- mouse embryo fibroblasts. Oncogene. 13:739-748.

16. Yu, D., and Thomas-Tikhonenko, A. 2002. A nontransgenic mouse model for B-cell lymphoma: in vivo infection of p53-null bone marrow progenitors by a Myc retrovirus is sufficient for tumorigenesis. Oncogene. 21:1922-1927.

17. Yu, D., Dews, M., Park, A., Tobias, J.W., and Thomas-Tikhonenko, A. 2005. Inactivation of Myc in murine two-hit B lymphomas causes dormancy with elevated levels of interleukin 10 receptor and CD20: implications for adjuvant therapies. Cancer Res. 65:5454-5461.

18. Wiselogle, F.Y. 1946. A survey of antimalarial drugs, 1941-1945. J.W. Edwards. Ann Arbor, Michigan, USA. 134 pp.

19. Michihara, A., et al. 2005. Disruptive effect of chloroquine on lysosomes in cultured rat hepatocytes. Biol. Pharm. Bull. 28:947-951.

20. Fedorko, M.E., Hirsch, J.G., and Cohn, Z.A. 1968 Autophagic vacuoles produced in vitro. II. Studies on the mechanism of formation of autophagic vacuoles produced by chloroquine. J. Cell Biol. 38:392-402.

21. Boya, P., et al. 2005. Inhibition of macroautophagy triggers apoptosis. Mol. Cell. Biol. 25:1025-1040.

22. Tanida, I., Ueno, T., and Kominami, E. 2004. LC3 conjugation system in mammalian autophagy. Int. J. Biochem. Cell Biol. 36:2503-2518.

23. Mizushima, N., Yamamoto, A., Matsui, M., Yoshimori, T., and Ohsumi, Y. 2004. In vivo analysis of autophagy in response to nutrient starvation using transgenic mice expressing a fluorescent autophagosome marker. Mol. Biol. Cell. 15:1101-1111.

24. Mizushima, N., et al. 2001. Dissection of autophagosome formation using Apg5-deficient mouse embryonic stem cells. J. Cell Biol. 152:657-668.

25. Elmore, S.P., Qian, T., Grissom, S.F., and Lemasters, J.J. 2001. The mitochondrial permeability transition initiates autophagy in rat hepatocytes. FASEBJ 15:2286-2287

26. Lum, J.J., DeBerardinis, R.J., and Thompson, C.B
2005. Autophagy in metazoans: cell survival in the land of plenty. Nat. Rev. Mol. Cell Biol. 6:439-448.

27. Coiffier, B. 2005. State-of-the-art therapeutics: diffuse large B-cell lymphoma. J. Clin. Oncol. 23:6387-6393.

28. Witzig, T.E. 2005. Current treatment approaches for mantle-cell lymphoma. J. Clin. Oncol. 23:6409-6414.

29. Hiddemann, W., et al. 2005. Treatment strategies in follicular lymphomas: current status and future perspectives. J. Clin. Oncol. 23:6394-6399.

30. Kim, W.J., Beardsley, D.I., Adamson, A.W., and Brown, K.D. 2005. The monofunctional alkylating agent $\mathrm{N}$-methyl-N'-nitro- $\mathrm{N}$-nitrosoguanidine triggers apoptosis through p53-dependent and -independent pathways. Toxicol. Appl. Pharmacol. 202:84-98.

31. Prasad, R.N., Mahajan, R.C., and Ganguly, N.K. 1987. Effect of chloroquine on cellular immune responses of normal and P. knowlesi-infected rhesus monkeys. Immunol. Cell Biol. 65:211-216.

32. Yin, F., Guo, M., and Yao, S. 2003. Kinetics of DNA binding with chloroquine phosphate using capacitive sensing method. Biosens. Bioelectron. 19:297-304.

33. Aita, V.M., et al. 1999. Cloning and genomic organization of beclin 1, a candidate tumor suppressor gene on chromosome 17q21. Genomics. 59:59-65.

34. Liang, X.H., et al. 1999. Induction of autophagy and inhibition of tumorigenesis by beclin 1. Nature. 402:672-676

35. Gu, Y., Wang, C., and Cohen, A. 2004. Effect of IGF-1 on the balance between autophagy of dysfunctional mitochondria and apoptosis. FEBS Lett. 577:357-360

36. Iwata, J.I., et al. 2005. Excess peroxisomes are degraded by autophagic machinery in mammals. J. Biol. Chem. 281:4035-4041.

37. Kissova, I., Deffieu, M., Manon, S., and Camougrand, N. 2004. Uth1p is involved in the autophagic degradation of mitochondria. J. Biol. Chem. 279:39068-39074.

38. Bandara, P.D., Flattery-O'Brien, J.A., Grant, C.M., and Dawes, I.W. 1998. Involvement of the Saccharomyces cerevisiae UTH1 gene in the oxidativestress response. Curr. Genet. 34:259-268.

39. Gorka, M., et al. 2005. Autophagy is the dominant type of programmed cell death in breast cancer MCF-7 cells exposed to AGS 115 and EFDAC, new sesquiterpene analogs of paclitaxel. Anticancer Drugs. 16:777-788.

40. Gozuacik, D., and Kimchi,A. 2004. Autophagy as a cell death and tumor suppressor mechanism. Oncogene. 23:2891-2906
41. Boxer, L.M., and Dang, C.V. 2001. Translocations involving c-myc and c-myc function. Oncogene. 20:5595-5610.

42. Little, C.D., Nau, M.M., Carney, D.N., Gazdar, A.F., and Minna, J.D. 1983. Amplification and expression of the c-myc oncogene in human lung cancer cell lines. Nature. 306:194-196.

43. Mariani-Costantini, R., et al. 1988. In situ c-myc expression and genomic status of the c-myc locus in infiltrating ductal carcinomas of the breast. Cancer Res. 48:199-205.

44. Augenlicht, L.H., et al. 1997. Low-level c-myc amplification in human colonic carcinoma cell lines and tumors: a frequent, p53-independent mutation associated with improved outcome in a randomized multi-institutional trial. Cancer Res. 57:1769-1775.

45. Hemann, M.T., et al. 2005. Evasion of the p53 tumour surveillance network by tumour-derived MYC mutants. Nature. 436:807-811.

46. Erisman, M.D., et al. 1985. Deregulation of c-myc gene expression in human colon carcinoma is not accompanied by amplification or rearrangement of the gene. Mol. Cell. Biol. 5:1969-1976.

47. Escot, C., et al. 1986. Genetic alteration of the c-myc protooncogene (MYC) in human primary breast carcinomas. Proc. Natl. Acad. Sci. U. S. A. 83:4834-4838.

48. Dang, C.V. 1999. c-Myc target genes involved in cell growth, apoptosis, and metabolism. Mol. Cell. Biol. 19:1-11.

49. Degenhardt, K., et al. 2006. Autophagy promotes tumor cell survival and restricts necrosis, inflammation, and tumorigenesis. Cancer Cell. 10:51-64.

50. O’Neill, P.M., Bray, P.G., Hawley, S.R., Ward, S.A., and Park, B.K. 1998. 4-Aminoquinolines-past, present, and future: a chemical perspective. Pharmacol. Ther. 77:29-58.

51. Kremer, J.M. 2001. Rational use of new and existing disease-modifying agents in rheumatoid arthritis. Ann. Intern. Med. 134:695-706.

52. Walenta, S., Schroeder, T., and Mueller-Klieser, W. 2004. Lactate in solid malignant tumors: potential basis of a metabolic classification in clinical oncology. Curr. Med. Chem. 11:2195-2204.

53. Carmichael, S.J., Charles, B., and Tett, S.E. 2003. Population pharmacokinetics of hydroxychloroquine in patients with rheumatoid arthritis. Ther. Drug Monit. 25:671-681.

54. Munster, T., et al. 2002. Hydroxychloroquine concentration-response relationships in patients with rheumatoid arthritis. Arthritis Rheum. 46:1460-1469. 